\title{
BMJ Open Effects of computerised clinical decision support systems (CDSS) on nursing and allied health professional performance and patient outcomes: a systematic review of experimental and observational studies
}

\author{
Teumzghi F Mebrahtu (1) , ${ }^{1}$ Sarah Skyrme, ${ }^{2}$ Rebecca Randell (I) ,3,4 \\ Anne-Maree Keenan, ${ }^{2}$ Karen Bloor (D) , ${ }^{5}$ Huiqin Yang, ${ }^{2}$ Deirdre Andre, ${ }^{6}$ \\ Alison Ledward, ${ }^{2}$ Henry King, ${ }^{2}$ Carl Thompson ${ }^{2}$
}

To cite: Mebrahtu TF, Skyrme S, Randell $\mathrm{R}$, et al. Effects of computerised clinical decision support systems (CDSS) on nursing and allied health professional performance and patient outcomes: a systematic review of experimental and observational studies. BMJ Open 2021;11:e053886. doi:10.1136/ bmjopen-2021-053886

- Prepublication history and additional supplemental material for this paper are available online. To view these files, please visit the journal online (http://dx.doi.org/10.1136/ bmjopen-2021-053886)

Received 26 May 2021 Accepted 22 November 2021

Check for updates

(C) Author(s) (or their employer(s)) 2021. Re-use permitted under CC BY. Published by BMJ.

For numbered affiliations see end of article.

Correspondence to Teumzghi F Mebrahtu; t.f.mebrahtu@leeds.ac.uk

\section{ABSTRACT}

Objective Computerised clinical decision support systems (CDSS) are an increasingly important part of nurse and allied health professional (AHP) roles in delivering healthcare. The impact of these technologies on these health professionals' performance and patient outcomes has not been systematically reviewed. We aimed to conduct a systematic review to investigate this. Materials and methods The following bibliographic databases and grey literature sources were searched by an experienced Information Professional for published and unpublished research from inception to February 2021 without language restrictions: MEDLINE (Ovid), Embase Classic+Embase (Ovid), PsycINFO (Ovid), HMIC (Ovid), AMED (Allied and Complementary Medicine) (Ovid), CINAHL (EBSC0), Cochrane Central Register of Controlled Trials (Wiley), Cochrane Database of Systematic Reviews (Wiley), Social Sciences Citation Index Expanded (Clarivate), ProQuest Dissertations \& Theses Abstracts \& Index, ProQuest ASSIA (Applied Social Science Index and Abstract), Clinical Trials.gov, WHO International Clinical Trials Registry (ICTRP), Health Services Research Projects in Progress (HSRProj), OpenClinical(www.OpenClinical. org), OpenGrey (www.opengrey.eu), Health.IT.gov, Agency for Healthcare Research and Quality (www.ahrq.gov). Any comparative research studies comparing CDSS with usual care were eligible for inclusion.

Results A total of 36106 non-duplicate records were identified. Of 35 included studies: 28 were randomised trials, three controlled-before-and-after studies, three interrupted-time-series and one non-randomised trial. There were $\sim 1318$ health professionals and $\sim 67595$ patient participants in the studies. Most studies focused on nurse decision-makers $(71 \%)$ or paramedics $(5.7 \%)$. CDSS as a standalone Personal Computer/LAPTOP-technology was a feature of $88.7 \%$ of the studies; only $8.6 \%$ of the studies involved 'smart' mobile/handheld-technology. Discussion CDSS impacted $38 \%$ of the outcome measures used positively. Care processes were better in $47 \%$ of the measures adopted; examples included,
Strengths and limitations of this study

The review is based on a comprehensive literature search.

- This is the first systematic review of clinical decision support systems influence on nursing and allied health professional (AHP) performance and outcomes.

- AHPs are under-represented, with a primary focus on paramedics and physiotherapists.

- The number of studies, service users/patients and health professionals involved was sizeable, but outcomes were too heterogeneous to aggregate.

- The overall quality of comparative research represented by the included studies was poor.

nurses' adherence to hand disinfection guidance, insulin dosing, on-time blood sampling and documenting care. Patient care outcomes in $40.7 \%$ of indicators were better; examples included, lower numbers of falls and pressure ulcers, better glycaemic control, screening of malnutrition and obesity and triaging appropriateness.

Conclusion CDSS may have a positive impact on selected aspects of nurses' and AHPs' performance and care outcomes. However, comparative research is generally low quality, with a wide range of heterogeneous outcomes. After more than 13 years of synthesised research into CDSS in healthcare professions other than medicine, the need for better quality evaluative research remains as pressing.

\section{INTRODUCTION}

Nurses and allied health professionals' (AHPs') judgements and decisions commit financial, human and technical resources to care in health systems. ${ }^{1}$ To support decisionmaking and underpin new roles and ways of delivering services, such as nurse-led 
primary care, ${ }^{1}$ computerised clinical decision support systems (CDSS) have been developed to tailor evidencebased advice provided to clinicians at the point of decision-making.

CDSS can improve professional performance by making the basis for decisions explicit; widening available information, encouraging more consistent decisions and thus reducing unwarranted variation in processes and patient outcomes. ${ }^{2}$ Negatively, CDSS could encourage a focus on unimportant problems, hinder care delivery and contribute to a widening of (digital) inequalities. ${ }^{4-6}$

Reviews focusing mainly on doctors suggest CDSS effects on performance and outcomes are inconsistent, ${ }^{7}$ but improved care processes ${ }^{89}$ and reduced morbidity ${ }^{8}$ and mortality ${ }^{10}$ are possible. These reviews, however, often neglect the multidisciplinary nature of healthcare delivery and the decisions involved.

Previously synthesised studies of nurses' use of CDSS suggest only limited impact on performance and health outcomes. ${ }^{11}$ Digital technology and research evidence have both developed significantly since this review was undertaken. In this review, we aim to examine the impact of CDSS on nurses' and allied health professionals' (AHPs) performance and patient outcomes.

\section{REVIEW METHODS}

Following best practice principles, ${ }^{12}{ }^{13}$ we undertook a systematic review of research into CDSS targeting nurse and AHP decision-makers. The protocol was registered with PROSPERO ${ }^{14}$ (number: CRD42019147773).

\section{Literature searching}

Initial searches were conducted in November 2019 and updated on 12 February 2021. Searches were not restricted by language. See online supplemental table 1 for search terms.

We searched: MEDLINE(Ovid), Embase Classic+Embase (Ovid), PsycINFO (Ovid), Health Management Information Consortium (HMIC) (Ovid), AMED (Allied and Complementary Medicine) (Ovid), CINAHL, Cochrane Central Register of Controlled Trials (Wiley, Cochrane Database of Systematic Reviews (Wiley), Social Sciences Citation Index Expanded (Clarivate), ProQuest Dissertations and Theses Abstracts and Index, ProQuest ASSIA (Applied Social Science Index and Abstract), Clinical Trials.gov, WHO International Clinical Trials Registry (ICTRP), Health Services Research Projects in Progress (HSRProj), OpenClinical(www.OpenClinical.org), OpenGrey (www.opengrey.eu), Health.IT.gov, Agency for Healthcare Research and Quality (www.ahrq.gov).

\section{Study inclusion and exclusion}

All titles and abstracts were imported into a reference management database (EndNote) and duplicates removed. Covidence review production toolkit (www. covidence.org) was used to manage screening, data extraction and organising of the review and ensure efficient production. After removing duplicate titles and abstracts, seven reviewers (A-MK, CT, HY, HK RR, SS and TFM) independently screened all titles and abstracts. TFM first-screened titles and abstracts for all studies, the other six authors then second-screened $16.7 \%$ of the studies each. Records with decision disagreements were revisited by two authors (TFM and CT) and resolved by consensus, a third reviewer (RR) was available for further disagreements although none occurred. Two reviewers (CT and TFM) independently assessed study relevance using Cochrane Collaboration's Effective Practice and Organisation of Care (EPOC) criteria; ${ }^{15}$ and, conducted full-text screening. Any disagreements were resolved by consensus.

Comparative studies (randomised controlled trials (RCTs), non-randomised trials, controlled before-after (CBA) studies, interrupted time series (ITS) studies and repeated measures studies) comparing CDSS against usual care (ie, clinical decision-making unsupported by CDSS) were eligible for inclusion.

\section{Participants}

Studies that evaluate the effects of CDSS used by nurses (including midwives) and AHPs and report professional performance and patient outcomes were eligible for inclusion.

\section{Interventions}

The eligible intervention in this review was the use of any form of CDSS to aid clinical decision making.

\section{Comparator}

The comparator was usual care, defined as clinical practice where clinical decision making is unsupported by CDSS.

\section{Outcomes}

Our primary outcome was adherence of nurses and AHPs to evidence-based recommendations. Secondary outcomes were diagnostic accuracy, time to reach judgement, adverse events, health professional satisfaction and system and/or implementation costs and benefits.

\section{Data extraction}

Data on study characteristics and outcomes were independently extracted by two reviewers (CT and TFM) using the EPOC standard data collection form. ${ }^{16}$

\section{Quality assessment}

Study quality and risk of bias was assessed independently by CT and TFM using Cochrane Handbook for Systematic Reviews of Interventions ${ }^{17}$ and EPOC guidelines. ${ }^{18}$

Each potential source of bias was judged as high, low or unclear, and an overall 'risk of bias' classification (high, moderate or low) assigned to each included study. ${ }^{17}$ Studies with low risk of bias in all domains, or where bias was unlikely to fundamentally alter results, were treated as low risk. Studies with bias risk in at least one domain, or where bias might alter conclusions, were treated as unclear. Studies with a high risk of bias in at least one 


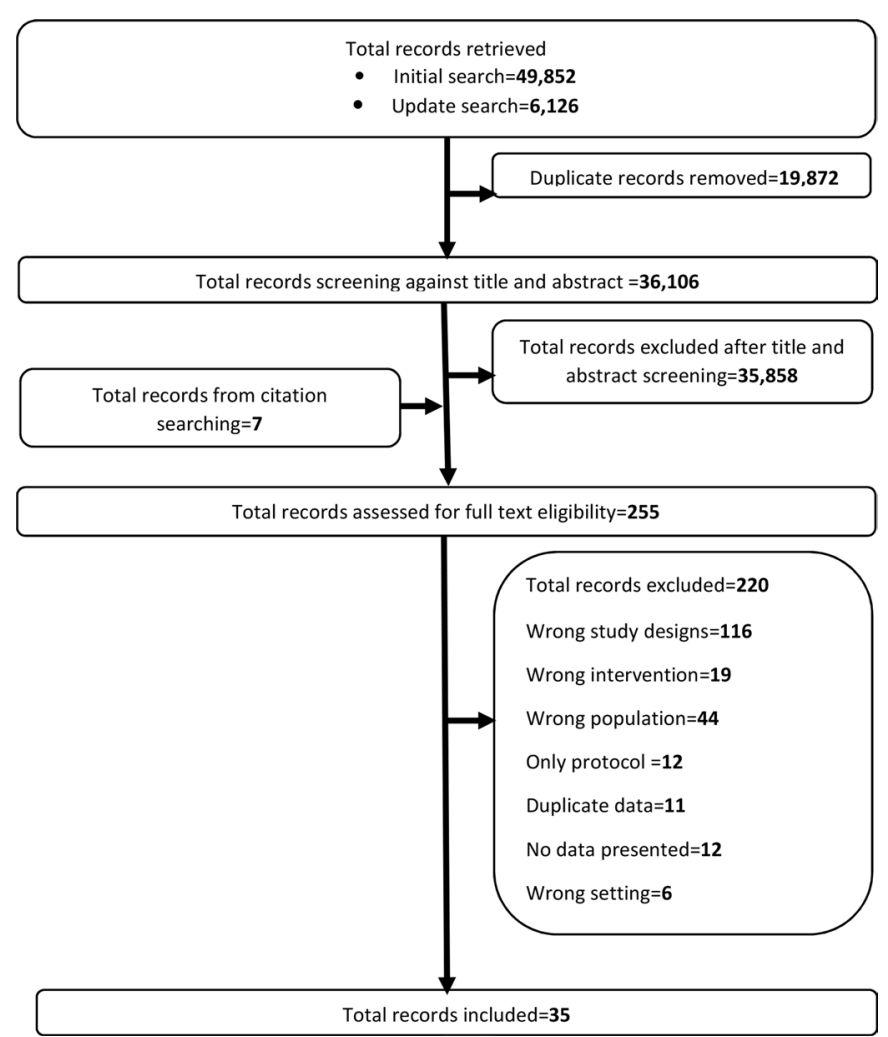

Figure 1 PRISMA flow chart of study selection process. PRISMA, Preferred Reporting Items for Systematic Reviews and Meta-Analyses.

domain, or with a serious bias likely to reduce the certainty of conclusions, were considered high risk.

\section{Data synthesis}

Findings were synthesised narratively, regardless of statistical analysis in the primary study. Studies were grouped by (i) similarity in focus or CDSS-type (knowledge based or machine learning), (ii) health professionals targeted, (iii) patient group, (iv) outcomes reported and (v) study design.

If not reported, we calculated absolute risks from the primary research. Risk differences and $95 \%$ CIs were then calculated from these. Because the CDSS, participants and underlying research questions were so heterogeneous no meta-analysis was undertaken. ${ }^{19}$

\section{RESULTS}

\section{Evidence quantity}

From 36106 non-duplicate records identified, 35858 records were excluded after title and abstract screening. Seven records were identified through forward citation searching. Full-text screening was undertaken on 255 records which led to 220 more records being excluded. Thirty-five studies were included in the review. ${ }^{20-51}$ Figure 1 illustrates study selection.

\section{Study descriptions}

The 35 included studies comprised 28 RCTs (80\%), three CBA studies $(8.6 \%)$, three ITS $(8.6 \%)$ and one non-randomised trial (2.8\%). Thirty-two studies $(91.4 \%)$ were peer-reviewed journal articles and three $(8.6 \%)$ were $\mathrm{PhD}$ theses. The public sector funded $74.3 \%$ of studies; industry, $5.7 \% ; 17.1 \%$ failed to declare funding and $2.9 \%$ were unfunded. Most studies were published after 2010 $(\mathrm{n}=29,82.9 \%)$ with just two studies during 1997-1999 and $14(40.0 \%)$ in 2000-2010. Sixteen studies (45.6\%) were published after the last significant systematic review on CDSS for nurses' performance and health outcomes. ${ }^{11}$ Circa 1318 health professionals and 67595 patients were study participants, mainly in hospital-based studies (57.1\%). Primary care accounted for $17.1 \%$ and nursing homes $11.4 \%$ of studies. Western health systems provided the dominant context: US (28.6\%); UK (20.0\%), Netherlands (17.2\%), Czech Republic and Norway (5.7\%) each; with single study representation (2.8\%) from Belgium, Brazil, China, Ghana, Norway, Sweden, Turkey and one multicentre (Austria, Czech Republic, and UK) report. See table 1.

Only one study (of 35) reported explicit theory to guide implementation of the CDSS. Almost a third (28\%) published their study protocol—none of which discussed theory-influenced implementation.

Nurses made up the target for the CDSS and control groups in $25(71.4 \%)$ studies; paramedics in two (5.7\%) studies. Five studies $(14.3 \%)$ compared nurses in the intervention (CDSS) group with physicians in the control. Two studies $(5.7 \%)$ recruited a combination of nurses and physiotherapists for CDSS and control groups. Thirty-one studies $(88.7 \%$ ) used a standalone (physically, even when integrated in an electronic health record) computerbased CDSS; three (8.6\%) used handheld/mobile-based technologies and just one study $(0.2 \%)$ used a web-based CDSS. CDSS were mostly designed with a single function in mind (eg, disease diagnosis), but some addressed multiple parts of clinical pathways (eg, disease diagnosis and disease management).

\section{Quality of identified evidence}

Except for three RCTs scored as 'Unclear', all studies were at 'high' overall risk of bias. On average, RCTs scored 'Low' risk of bias in five of nine domains; CBA studies were lower, with four domains; non-randomised studies scored 'low' for a single domain. The three ITS studies were 'Low' risk of bias in six (of seven) domains. Evidence quality did not change over time (see online supplemental table 2).

\section{Effects of intervention}

Most studies reported more than two outcomes from a total of 124 individual outcomes reported (115 distinct types of measured outcomes). There were five distinct outcome groups:

- Care processes: aspects of patient data collection and management, and the process of patient management.

- Care outcomes: patient health outcomes (eg, fall and pressure ulcer prevention rate). 
Table 1 Baseline characteristics of included studies

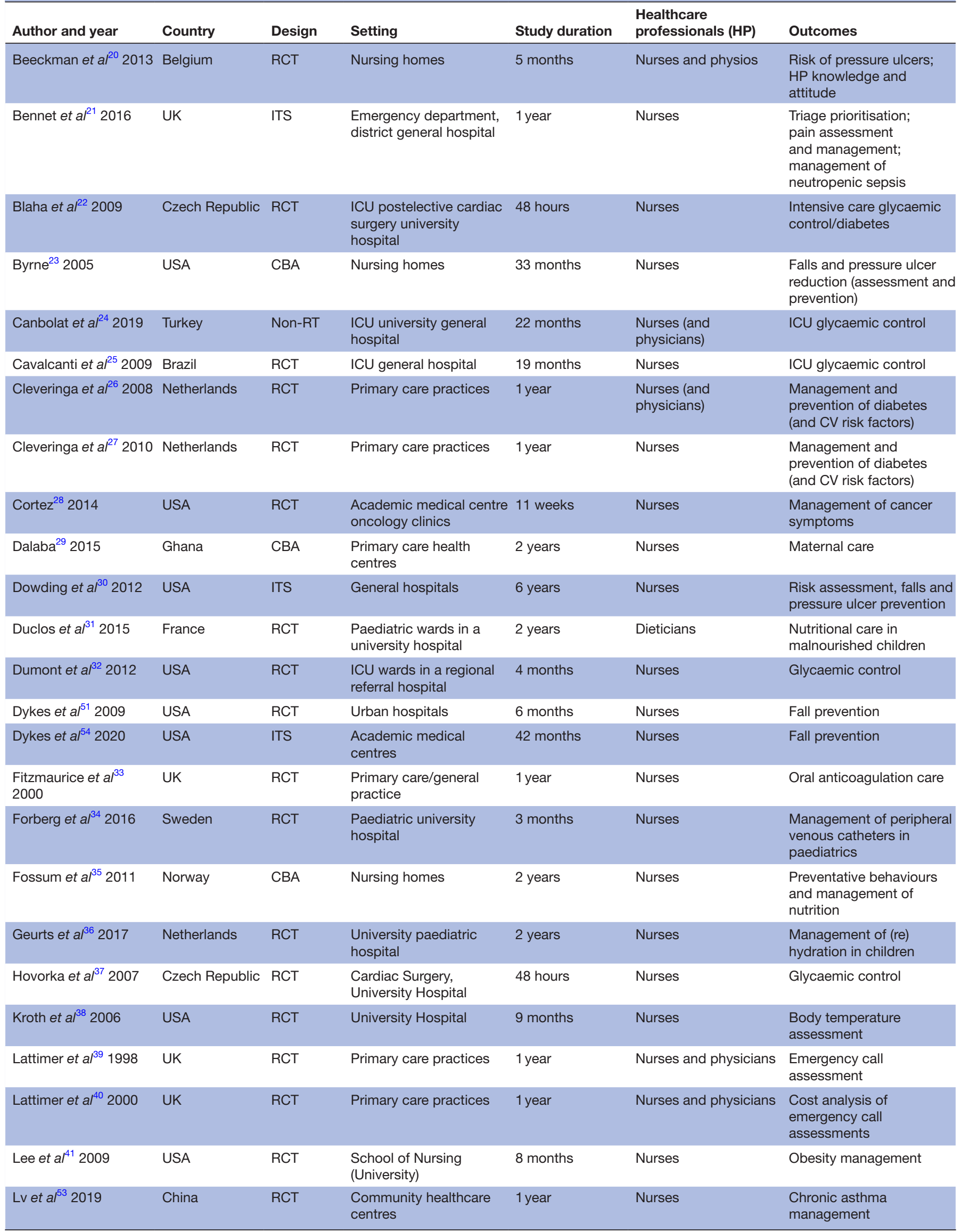


Table 1 Continued

\begin{tabular}{|c|c|c|c|c|c|c|}
\hline Author and year & Country & Design & Setting & Study duration & $\begin{array}{l}\text { Healthcare } \\
\text { professionals (HP) }\end{array}$ & Outcomes \\
\hline Mann et al ${ }^{42} 2011$ & USA & $\mathrm{RCT}$ & $\begin{array}{l}\text { Surgical Military hospital } \\
\text { ICU }\end{array}$ & 6 days & Nurses & $\begin{array}{l}\text { Glycaemic control in burn } \\
\text { intensive care patients }\end{array}$ \\
\hline McDonald et al ${ }^{43} 2017$ & USA & $\mathrm{RCT}$ & Nursing care homes & 2 months & Nurses & $\begin{array}{l}\text { Management of chronic } \\
\text { medical condition }\end{array}$ \\
\hline Paulson et a/52 2020 & Norway & $\mathrm{RCT}$ & University hospital & 10 months & Nurses & $\begin{array}{l}\text { Management of } \\
\text { malnutrition }\end{array}$ \\
\hline Rood et al ${ }^{45} 2005$ & Netherlands & $\mathrm{RCT}$ & $\begin{array}{l}\text { Surgical ICU in a } \\
\text { teaching hospital }\end{array}$ & 10 weeks & Nurses & Glycaemic control \\
\hline Roukema et al ${ }^{46} 2008$ & Netherlands & $\mathrm{RCT}$ & Children's Hospital & 27 months & Nurses & $\begin{array}{l}\text { Management of children } \\
\text { with fever without } \\
\text { apparent source }\end{array}$ \\
\hline Vadher et a/9 1997 & UK & $\mathrm{RCT}$ & $\begin{array}{l}\text { Cardiovascular medicine, } \\
\text { general hospital }\end{array}$ & & $\begin{array}{l}\text { A nurse and Trainee } \\
\text { doctors }\end{array}$ & Oral anticoagulant control \\
\hline Wells $^{50} 2013$ & UK & $\mathrm{RCT}$ & $\begin{array}{l}\text { Emergency ambulance } \\
\text { services }\end{array}$ & 1 year & Paramedics & $\begin{array}{l}\text { Emergency fall } \\
\text { assessment and } \\
\text { management }\end{array}$ \\
\hline
\end{tabular}

CBA, controlled before and after; ICU, intensive care unit; ITS, interrupted time-series; RCT, randomised controlled trials.

- Health professionals' knowledge, beliefs and behaviours: outcomes that relate to the health professionals themselves (eg, changed attitude and perception due to CDSS use).

- Adverse events: safety issues that could arise due to the use of CDSS (eg, morbidity).

- Economic costs and consequences: outcomes that relate to direct costs, savings, or cost-effectiveness of CDSS.

\section{Care process}

CDSS was better than usual care for 16 of $34(47.0 \%)$ care process outcomes. Care delivery was worse $(\mathrm{n}=5,14.7 \%)$ or no different for $13(38.2 \%)$ processes. See online supplemental table 3 .

\section{Adherence to guidelines}

The four RCTs reporting nurses' adherence to guidelines examined 10 outcomes. ${ }^{32} 344549$ Only one trial reported baseline and follow-up data for both arms,${ }^{34}$ CDSS users had better adherence to hand disinfection guidelines (risk difference $=6.7 \%$; $95 \%$ CI: $4.9 \%$ to $8.5 \%$ ); but were less likely to follow guidelines on disposable glove use (risk difference $=-1.4 \%$; 95\% CI: -2.2 to $-0.5 \%$ ) and daily inspections of Peripheral Venous Catheters (risk difference $=-5.2 \%$; $95 \%$ CI: -7.2 to $-3.3 \%$ ).

Two trials ${ }^{32} 45$ showed nurses using CDSS had better compliance with guidelines on insulin dosing (risk difference $=22 \%$; $95 \%$ CI: $19 \%$ to $25 \%$ ) and on-time blood sampling (risk difference $=4.7 \%$; $95 \%$ CI: $2.0 \%$ to $7.4 \%$ ). They deviated less from protocols (mean score difference out of $10=-2.6 ; 95 \%$ CI: -4.5 to -0.71$)$ and concurred more with recommended insulin doses (than trainee doctors). ${ }^{49}$

Patient assessment, diagnosis and treatment practices

Five RCTs ${ }^{31} 36384650$ and one ITS $^{21}$ reported 18 indicators of patient assessment and treatment quality. Pain assessment quality (pain score use and appropriateness of choices) of emergency department patients improved by $62.7 \%$ (95\% CI: $59.6 \%$ to $65.8 \%$ ) and investigation of inpatient paediatric malnutrition aetiology was $21.2 \%$ higher (95\% CI: $15.9 \%$ to $26.5 \%$ ) with CDSS. However, optimal IV antibiotics administration for sepsis was lower reduced by $5.9 \%$ (95\% CI: -8.3 to -3.5 ). Laboratory tests (electrolytes level acid-base balance test) and nutrition supplements (oral Rehydration Solution and intravenous rehydration) were no more likely to be ordered for paediatric inpatients by CDSS-enabled nurses.

There were marginally fewer wrongly recorded temperatures in hospital inpatients among CDSS-enabled nurses (risk difference $=-0.8 \%, 95 \%$ CI: -0.9 to -0.6 ). Vital signs recording in patients attended by paramedics were also not significantly different.

\section{Documenting care}

One ITS and a randomised trial reported five documentation-focused indicators. ${ }^{30}{ }^{52}$ Falls (risk ratio $=1.4,95 \%$ CI: 0.03 to 73.7$)$ and hospital acquired pressure ulcer risk assessments (risk ratio $=9.1,95 \%$ CI: 1.95 to 42.5 ) were higher with CDSS. As was nutritional 
care planning, food and fluid intake recording and treatment by nurses. ${ }^{52}$

\section{Referrals}

Paramedics using CDSS were more likely to refer patients to a community falls than send them to the emergency department (risk difference $=4.7 \%, 95 \%$ CI: 1.1 . to 8.3 ) ${ }^{48}$

\section{Patient care outcomes}

CDSS improved patient care outcomes in 22 of 54 (40.7\%) indicators and worsened them for one outcome indicator $(2.0 \%)$. See online supplemental table 4 .

\section{Blood glucose control}

Six RCTs 222526374244 and one non-randomised trial ${ }^{24}$ reported 19 indicators of glycaemic control, but only two reported baseline and follow-up values. ${ }^{22}{ }^{26}$ Blood glucose levels were better managed by ICU nurses using CDSS (mean=-2.2, $\mathrm{SD}=1.12$ ) compared with paper-based Mathias (mean=-1.2, $\mathrm{SD}=0.66$ ) and Bath (mean $=-1.5, \mathrm{SD}=0.78)$ protocols. $^{22}$ Glycated haemoglobin (A1C) $<7 \%$, systolic blood pressure $<140$ and total cholesterol $<4.5 \mathrm{mmol} / \mathrm{L}$ were higher by $4.6 \%$ (95\% CI: 2.7 to 6.5$), 10.2 \%$ (95\% CI: 7.9 to 12.5$)$ and $3.7 \%(95 \%$ CI: 1.2 to 6.2 ), respectively, in patients receiving care from CDSS-enabled nurses compared.

Trials reporting only follow-up data suggest better blood glucose control by CDSS-using nurses across a range of indicators: proportion in target range (risk difference $=32.9 \%$; 95\% CI: 20.0 to 46.0), occasions within the target glycaemic range $(80-110 \mathrm{mg} / \mathrm{dL}$ ) (risk difference $=33.0 \%, 95 \%$ CI: 20.5 to 45.4$)$, occasions over the target glycaemic range $(>110 \mathrm{mg} / \mathrm{dL})$ (risk difference $=-31.0 \%, 95 \%$ CI: -43.7 to -18.2$)$ and improvement of glycaemic control for 48 hours (risk difference $=40.0 \%$, 95\% CI: 27.4 to 52.6 )

\section{Blood coagulation management}

One RCT reported three indicators of blood coagulation management in primary care. ${ }^{33}$ Nurses using CDSS had significantly more tests in range (risk difference $=4.0 \%$, 95\% CI: 0.4 to 7.6 ) than doctors without CDSS. However, the improvement from baseline was lower among nurses (risk difference $=-1.9 \%$ (95\% CI: -3.1 to -0.7$)$, 'International Normalised Ratio (INR) Results within Range Point Prevalence' were not significantly different between the two groups and again, nurses using CDSS improved less than physicians without CDSS (risk difference $=-2.6 \%$, $95 \%$ CI: -5.3 to -0.1 ). There was no significant difference between groups in 'Time Spent within INR Target Range' (risk difference $=7.0 \%, 95 \% \mathrm{CI}:-0.7$ to 14.7 ).

\section{Antenatal and peripartum care}

The CBA study examining antenatal and peripartum care in community settings ${ }^{29}$ suggested CDSS-using midwives reduced delivery complications (per 1000 attendances) compared with usual care (risk difference $=2.4 \%, 95 \% \mathrm{CI}$ : 1.1 to 3.7$)$.
Managing patients with chronic comorbid diseases

Two RCTs examined three indicators of successfully managing patients with complex chronic multimorbid health conditions in care homes, ${ }^{43}$ and with asthma ${ }^{53}$ showed no significant differences between CDSS users and non-users for emergency room usage, hospitalisation and complexity of medication regimens.

\section{Obesity screening}

The RCT examining outpatient obesity screening by trainee nurses found CDSS-users had more 'encounters with obesity-related diagnosis' (risk difference $=10.3 \%$, 95\% CI: 8.0 to 12.5 ) and fewer 'encounters with missed obesity-related missed diagnosis' (risk difference $=41.0 \%$, 95\% CI: 48.8 to 35.0 ) than trainee nurses without CDSS. ${ }^{41}$

\section{Fall and pressure ulcer prevention and management}

Two RCTs, ${ }^{2051}$ two CBA studies ${ }^{2335}$ and two ITS ${ }^{3054}$ focused on fall or pressure ulcer prevention and management. In a single trial, ${ }^{20}$ pressure ulcer prevalence decreased more during the CDSS-enabled follow-up period (risk difference $=-6.3 \%, 95 \% \mathrm{CI}:-10.2$ to -2.4 ), a result which was reversed in one of the CBA studies (risk difference $=4.2 \%$, 95\% CI: 0.2 to 8.2$){ }^{35}$ The other CBA studies revealed no significant differences between CDSS using and nonusing nurses trying to prevent falls and pressure ulcers. ${ }^{23}$ In the ITS study, fall rate (risk ratio $=0.91,95 \% \mathrm{CI}: 0.75$ to 1.12) and hospital acquired pressure ulcer occurrence (risk ratio $=0.47,95 \%$ CI: 0.25 to 0.85 ) were significantly lower with CDSS. ${ }^{30}$

\section{Triage}

Three RCTs ${ }^{39} 4048$ and one ITS study ${ }^{21}$ evaluated CDSS impact on triage judgements. Health professionals using CDSS made fewer calls to general practitioners (GP) for telephone advice (risk difference $=-34.2 \%, 95 \%$ CI: -36.0 to -33.0 ), had fewer patients visited at home by duty GPs (risk difference $=-5.5 \%, 95 \%$ CI: -6.9 to -4.2 ) and fewer hospital admissions within 3 days (risk difference $=-0.98 \%$, $95 \%$ CI: -1.8 to -0.2$)$ of the judgement. There were no differences in, 'patients left at scene without conveyance to emergency department' (risk difference $=5.2 \%, 95 \%$ CI: -1.7 to 12.1$)$. The ITS study reported the proportion of correct (sic) triage prioritisation judgements was higher among CDSS-users (risk difference=24.7\%; 95\% CI: 18.8 to 30.6$)$.

\section{Quality of life and patients' satisfaction}

Two RCTs examined CDSS impact on quality of life and patient satisfaction. ${ }^{27} 48$ Patients in CDSS-using groups gained more life years (average difference in years $=0.14$, $95 \%$ CI: -0.12 to 0.40 ), more healthy years (average difference in years $=0.04,95 \% \mathrm{CI}:-0.07$ to 0.14 ) but reported lower quality of life and satisfaction. None of these differences were statistically significant.

Health professionals' knowledge, beliefs, and behaviour CDSS effects on knowledge, beliefs and behaviours of health professionals 20283247 were the focus of four RCTs 
using 12 indicators. CDSS increased 'Positive knowledge change' (risk difference $=6.5 \%$; 95\% CI: 0.8 to 13.2 ), 'positive attitude change' ( risk difference $=12.7 \%, 95 \%$ CI: 5.9 to 19.5), 'research utilisation' (risk difference $=9 \%$; $95 \%$ CI: 3.3 to 14.7 ), nurses' satisfaction (difference in satisfaction out of $10=3.6,95 \%$ CI: 2.4 to 4.8 ) and perceived deviations from protocols (mean difference out of $10=-4.7,95 \% \mathrm{CI}$ : -6.1 to -3.3$)$. Conversely, there was no significant impact on behaviours, intentions, perceived behavioural control, subjective and moral norms, barriers and research utilisation of CDSS-using nurses and physiotherapists (online supplemental table 5).

\section{Adverse events}

CDSS are not risk free, and three RCTs ${ }^{27} 33$ used four indicators to examine adverse events. Cardiovascular events in patients with diabetes (risk difference $=-11.0 \%$, 95\% CI: -18.0 to -4.0 ) and deaths in primary care patients (risk difference $=-5.7 \%, 95 \%$ CI: -10.1 to -1.7 ) were lower in CDSS-using groups of professionals. Serious adverse reactions in primary care patients and deaths in patients recently fallen and attended by paramedics were no less likely (online supplemental table 6).

\section{Economic costs and consequences}

Four RCTs ${ }^{27} 364048$ used 20 indicators to report economic costs and consequences of CDSS. Costs of managing cardiovascular disease were lower in CDSS users (cost difference $=-€ 587.00,95 \%$ CI: -880.00 to -294.00 ). Diabetes care cost more (cost difference $=€ 326.00,95 \%$ CI: 315.00 to 318.00 ); took longer per care task ('mean length of job cycle time' difference in minutes $=8.9$; $95 \%$ CI: 2.3 to $15.3)$ to generate an additional quality adjusted life-year (QALY) costing €38243.00 (online supplemental table $7)$.

\section{DISCUSSION}

\section{Summary of main results}

Our systematic review suggests that CDSS may improve some aspects of nurses' and AHPs' performance and care outcomes. Thirty-eight percent $(38 \%)$ of indicators were better. Of 35 included studies, 26 (74.3\%) reported CDSS-influenced care as better than care without CDSS on at least one outcome. In contrast, eight studies (22.8\%) showed no significant difference between CDSS and usual care, with seven studies suggesting CDSS were less effective than usual care for at least one outcome.

\section{Care processes}

Processes of care were better if CDSS was in use in almost half the studies, 16 of 34 (47\%); a headline that masks a very wide range of absolute improvement: from $0.7 \%$ to $62.7 \%$. Hand disinfection protocol adherence, insulin dosing, blood sampling at the right time and documented care were all better in CDSS users. This should be contrasted with the five $(16.1 \%)$ outcomes where CDSS provided no advantages over usual care. Both sets of findings are mitigated further by the considerable uncertainty in trying to estimate a holistic picture: the effects in 13 care process indicators $(41.9 \%)$ were not estimable; either because studies lacked power (lower than minimum acceptable of $80 \%$ ) to detect a difference in the comparison groups, or appropriate confidence intervals were not reported or could not be calculated from information published.

\section{Patient care outcomes}

CDSS was associated with significantly better patient care outcomes across a broad range of 22 of $54(40.7 \%)$ indicators (absolute difference between $4.6 \%$ and $42.9 \%$ ). Just one indicator (1.8\%) suggested no significant difference. Nurses using CDSS had better blood glucose control in emergency care patients (in five out of seven studies involved) and nurses and physiotherapists using CDSS were associated with better fall risk and pressure ulcer management. Triage was improved in nurses using CDSS in emergency call centres and paramedics faced with 'emergency falls' in older patients.

Health professionals' knowledge, beliefs, and behaviour

Improved knowledge, beliefs and behaviour occurred in three of 12 indicators (25\%). Nurse and physiotherapist CDSS-users had more knowledge and better attitudes compared with non-users. Compared with usual care, nurses utilised more research, were more satisfied at work, and perceived a greater need to follow protocols if they used CDSS.

\section{Adverse events}

CDSS generated fewer adverse events across two of four indicators (50\%). CDSS-using nurses had fewer cardiovascular events and reported deaths in their primary care patients compare to similar patients seen by doctors not using CDSS.

\section{Economic costs and consequences}

CDSS did not significantly increase costs, or save money. Costs per QALY was $€ 38243.00$ in one study—higher than the widely accepted willingness-to-pay threshold of $€ 20000$ per QALY ${ }^{27}$ and the UK de facto threshold of $£ 30000$ per QALY to be considered cost-effective by the National Institute for Health and Care Excellence. ${ }^{55}$

\section{Comparison with other studies or reviews}

Only one previous review has examined the effects of CDSS on nursing performance and patient outcomes. ${ }^{11}$ Twenty new primary studies have been published since this review; but inconsistent outcomes and weaknesses in study designs and methods remain. Given the importance of implementation in effectiveness, it was noteworthy that most studies lacked a theoretical foundation for the implementation of CDSS. Similarly, many studies did not report using guidelines for designing, conducting/evaluating and reporting CDSS-use. Of 35 included studies, just one used an explicit implementation model/theory 
at design stage. ${ }^{20}$ None of the studies discussed their findings with reference to implementation science/theory.

In their review of 100 trials-principally with doctorsGarg $e t a l^{7}$ reported improved performance in $64 \%$ and better patient outcomes in $13 \%$ of studies. Our results suggest greater improvement may be possible for nursing work in particular $(47 \%$ of process indicators and $41 \%$ of outcomes). Garg et al transformed improvement into a binary (yes/no) indicator and did not quantify the outcome improvements-making the clinical significance of improvements hard to ascertain.

Bright $e t a l^{8}$ reviewed RCTs of CDSS with a range of health professional decision-makers (doctors, nurses and AHPs). They reported improvements in processes of care $(\mathrm{OR}=1.55,95 \%$ CI: 1.38 to 1.74$)$ and morbidity ( $\mathrm{RR}=0.88$, 95\% CI: 0.80 to 0.96 ), but no impact on mortality (OR $=0.79,95 \%$ CI: 0.54 to 1.15$)$ or safety/adverse events ( $\mathrm{RR}=1.01,95 \% \mathrm{CI}: 0.90$ to 1.14$)$. However, outcomes measured were too heterogeneous for meta-analysis. The criteria for comparison groups were relaxed; the 'intervention' sometimes included paper-based decision support and alternative CDSS systems were used as a comparator in some studies. Our review required there to be an indication for the use of CDSS and a comparator that ruled out CDSS-use as part of 'usual care'. While we found improvements are possible from CDSS, comparison with Bright $e t a l$ s findings would be unreliable.

Moja and colleagues' review of $18 \mathrm{RCTs}^{10}$ (including nurses and AHPs alongside doctors) found no significant difference in CDSS-attributable mortality $(\mathrm{RR}=0.96$, 95\% CI: 0.85 to 1.08 ) but lower morbidity ( $R R=0.82$, $95 \%$ CI: 0.68 to 0.99$)$. While mortality and morbidity findings are similar to ours, their use of CDSS in the primary study comparator groups, again makes comparisons unreliable.

A recent review of 115 trials of CDSS, with a mix of health professionals, reported process improvements of the order of $5.8 \%$ (95\% CI: $4.0 \%$ to $7.6 \%$ ) with CDSS. ${ }^{9}$ As with Bright et al, the 'comparator' criteria were unclear and outcome measures too heterogeneous for metaanalysis. Studies with more than two comparators were treated as different trials, meaning double counting and multiple comparisons (p-hacking) could not be ruled out, confounding comparisons with our findings.

\section{Strengths and limitations}

Our review, while based on a comprehensive literature search, is a function of that literature. Consequently, we have highlighted primarily the impact of CDSS on nurses rather than AHPs. With the exception of paramedics and physiotherapists, other AHPs are poorly represented.

Evidence quality was poor and has not improved significantly since 2009. While the number of studies (35), service users/patients ( 67000) and health professionals ( 1318) involved were sizeable, outcomes were too heterogeneous for aggregation. Inconsistencies in the effects of CDSS on target health professionals' performance and patient outcomes remain unresolved.
Moreover, although we have used a comprehensive list of databases in our search, the possibility of missing studies due to search terms cannot be ruled-out.

\section{CONCLUSIONS}

CDSS can benefit nurse and (some) AHP delivered performance and patient outcomes. CDSS can improve adherence to guidelines and enhance patient care. Triaging of emergency patients, glycaemic control and screening of malnutrition and obesity all represent appropriate targets for CDSS. These conclusions require cautious interpretation: they are based on mainly low-quality studies, with heterogeneous outcomes and indicators.

To improve the quality of studies and consistency of outcomes, future research should satisfy two key requirements. First, system designers and evaluators should consider appropriate implementation theory/models (examples include Normalisation Process Theory ${ }^{56}$ and the NASSS framework ${ }^{57}$ given the planned technology and associated work to encourage sustained adoption. Second, study reporting is varied, poor quality and lacking essential detail for implementation; guidelines for conducting and reporting CDSS should be a feature of the publication of findings. This would make synthesis easier and more informative. Guidelines for CDSS reporting in general already exist, it is difficult to conceive why they cannot be applied to nursing and AHP-focused CDSS. ${ }^{5859}$

\section{Author affiliations}

${ }^{1}$ School of Computing, University of Leeds, Leeds, UK ${ }^{2}$ School of Healthcare, University of Leeds, Leeds, UK

${ }^{3}$ Faculty of Health Studies, University of Bradford, Bradford, UK

${ }^{4}$ Wolfson Centre for Applied Health Research, Bradford, UK

${ }^{5}$ Department of Health Sciences, University of York, York, UK

${ }^{6}$ Library Services, University of Leeds, Leeds, UK

\section{Twitter Rebecca Randell @HClforHealthIT and Karen Bloor @KBloor}

Contributors A-MK, AL, CT, DA, HY, KB and RR contributed to conception of the review. DA conducted online database searches. A-MK, CT, HK, HY, RR, SS and TFM contributed to titles and abstracts screening. CT and TFM contributed to full-text screening, quality assessment and data extraction. TFM analysed and summarised data as well as produced the first draft of the manuscript. All authors have been involved in revising the work for important intellectual content and have approved the final version for publication. TFM had full access to all of the data in the study and take responsibility for the integrity of the data and the accuracy of the data analysis.

Funding The review is funded by the National Institute for Health Research Health Services Delivery and Research programme (award number: NIHR127926). The views expressed in this publication are those of the authors and not necessarily those of the NHS, the NIHR or the Department of Health.

\section{Competing interests None declared.}

Patient consent for publication Not required.

Ethics approval This study does not involve human participants.

Provenance and peer review Not commissioned; externally peer reviewed.

Data availability statement All data relevant to the study are included in the article or uploaded as supplementary information. Data used in the review are all available in this article and supplementary materials.

Supplemental material This content has been supplied by the author(s). It has not been vetted by BMJ Publishing Group Limited (BMJ) and may not have been peer-reviewed. Any opinions or recommendations discussed are solely those 
of the author(s) and are not endorsed by BMJ. BMJ disclaims all liability and responsibility arising from any reliance placed on the content. Where the content includes any translated material, BMJ does not warrant the accuracy and reliability of the translations (including but not limited to local regulations, clinical guidelines, terminology, drug names and drug dosages), and is not responsible for any error and/or omissions arising from translation and adaptation or otherwise.

Open access This is an open access article distributed in accordance with the Creative Commons Attribution 4.0 Unported (CC BY 4.0) license, which permits others to copy, redistribute, remix, transform and build upon this work for any purpose, provided the original work is properly cited, a link to the licence is given, and indication of whether changes were made. See: https://creativecommons.org/ licenses/by/4.0/.

\section{ORCID iDs}

Teumzghi F Mebrahtu http://orcid.org/0000-0003-4821-2304

Rebecca Randell http://orcid.org/0000-0002-5856-4912

Karen Bloor http://orcid.org/0000-0003-4852-9854

\section{REFERENCES}

1 Murdoch J, Barnes R, Pooler J, et al. The impact of using computer decision-support software in primary care nurse-led telephone triage: interactional dilemmas and conversational consequences. Soc Sci Med 2015;126:36-47.

2 Anderson JA, Willson P. Clinical decision support systems in nursing. 2008;26:151-8.

3 Cresswell K, Majeed A, Bates DW, et al. Computerised decision support systems for healthcare professionals: an interpretative review. Inform Prim Care 2012;20:115-28.

4 Bonney W. Impacts and risks of adopting clinical decision support systems. Efficient Decision Support Systems: Practice and Challenges in Biomedical Related Domain, 2011: 21-30.

5 Langhorne P, Ramachandra S. Organised inpatient (stroke unit) care for stroke: network meta-analysis. Cochrane Database of Systematic Reviews 2020;4.

6 Sintchenko V, Coiera E. Decision complexity affects the extent and type of decision support use. AMIA Annu Symp Proc 2006;2006:724-8.

7 Garg AX, Adhikari NKJ, McDonald H, et al. Effects of computerized clinical decision support systems on practitioner performance and patient outcomes: a systematic review. JAMA 2005;293:1223-38.

8 Bright TJ, Wong A, Dhurjati R, et al. Effect of clinical decision-support systems: a systematic review. Ann Intern Med 2012;157:29-43.

9 Kwan JL, Lo L, Ferguson J, et al. Computerised clinical decision support systems and absolute improvements in care: meta-analysis of controlled clinical trials. BMJ 2020;370:m3216.

10 Moja L, Kwag KH, Lytras T, et al. Effectiveness of computerized decision support systems linked to electronic health records: a systematic review and meta-analysis. Am J Public Health 2014;104:e12-22.

11 Randell R, Mitchell N, Dowding D, et al. Effects of computerized decision support systems on nursing performance and patient outcomes: a systematic review. J Health Serv Res Policy 2007;12:242-51.

12 Centre for Reviews and Dissemination. Systematic Reviews: CRD's Guidance for Undertaking Reviews in Health Care. York: Centre for Reviews and Dissemination: University of York, 2009.

13 Liberati A, Altman DG, Tetzlaff J. The PRISMA statement for reporting systematic reviews and meta-analyses of studies that evaluate health care interventions: explanation and elaboration. Ann Intern Med 2009;151:W-65-W-94.

14 Thompson C, Randell R, Keenan AM. Effects of computerised clinical decision support systems (CDSS) on nursing and allied health professional performance and patient outcomes: a systematic review and user contextualisation.: Prospero, 2019. Available: https://www. crd.york.ac.uk/prospero/display_record.php?ID=CRD42019147773

15 Cochrane Effective Practice Organisation of Care (EPOC). Epoc resources for review authors: what study designs can be considered for inclusion in an EPOC review and what should that be called? 2017. Available: http://epoc.cochrane.org/resources/epoc-resourcesreview-authors [Accessed 15 Apr 2020].

16 Cochrane Effective Practice and Organisation of Care (EPOC). Data collection form. EPOC resources for review authors, 2017. Available: https://epoc.cochrane.org/resources/epoc-resources-review-authors [Accessed 30 Mar 2020].

17 Higgins JPT TJ, Chandler J, Cumpston M, eds. Cochrane Handbook for Systematic Reviews of Interventions version 6.0 (updated July 2019). Cochrane, 2019.
18 Cochrane Effective Practice and Organisation of Care (EPOC) Suggested risk of bias criteria for EPOC reviews. EPOC resources for review authors, 2017. Available: https://epoc.cochrane.org/ resources/epoc-resources-review-authors

19 Borenstein M, Hedges L, Higgins J. When does it make sense to perform a meta-analysis? introduction to meta-analysis. Chester, UK: John Wiley \& Sons Ltd, 2009.

20 Beeckman D, Clays E, Van Hecke A, et al. A multi-faceted tailored strategy to implement an electronic clinical decision support system for pressure ulcer prevention in nursing homes: a two-armed randomized controlled trial. Int J Nurs Stud 2013;50:475-86.

21 Bennett $\mathrm{P}$, Hardiker N. A quantitative study investigating the effects of computerised clinical decision support in the emergency department. Stud Health Technol Inform 2016;225:53-7.

22 Blaha J, Kopecky P, Matias M, et al. Comparison of three protocols for tight glycemic control in cardiac surgery patients. Diabetes Care 2009;32:757

23 Byrne CM. Impact of prospective computerized clinical decision support information and targeted assistance on nursing home resident outcomes. State University of New York at Albany, 2005.

24 Canbolat O, Kapucu S, Kilickaya O. Comparison of routine and computer-guided glucose management for glycemic control in critically ill patients. Crit Care Nurse 2019;39:20-7.

25 Cavalcanti AB, Silva E, Pereira AJ, et al. A randomized controlled trial comparing a computer-assisted insulin infusion protocol with a strict and a conventional protocol for glucose control in critically ill patients. J Crit Care 2009;24:371-8.

26 Cleveringa FGW, Gorter KJ, van den Donk M, et al. Combined task delegation, computerized decision support, and feedback improve cardiovascular risk for type 2 diabetic patients: a cluster randomized trial in primary care. Diabetes Care 2008;31:2273-5.

27 Cleveringa FGW, Welsing PMJ, van den Donk M, et al. CostEffectiveness of the diabetes care protocol, a multifaceted computerized decision support diabetes management intervention that reduces cardiovascular risk. Diabetes Care 2010;33:258-63.

28 Cortez S. Measuring active clinical decision support influence on nursing research utilization. University of Phoenix, 2014.

29 Dalaba MA, Akweongo P, Aborigo RA, et al. Cost-Effectiveness of clinical decision support system in improving maternal health care in Ghana. PLoS One 2015;10:e0125920.

30 Dowding DW, Turley M, Garrido T. The impact of an electronic health record on nurse sensitive patient outcomes: an interrupted time series analysis. J Am Med Inform Assoc 2012;19:615-20.

31 Duclos A, Touzet S, Restier L, et al. Implementation of a computerized system in pediatric wards to improve nutritional care: a cluster randomized trial. Eur J Clin Nutr 2015;69:769-75.

32 Dumont C, Bourguignon C. Effect of a computerized insulin dose calculator on the process of glycemic control. Am J Crit Care 2012;21:106-15.

33 Fitzmaurice DA, Hobbs FD, Murray ET, et al. Oral anticoagulation management in primary care with the use of computerized decision support and near-patient testing: a randomized, controlled trial. Arch Intern Med 2000;160:2343-8.

34 Förberg U, Unbeck M, Wallin L, et al. Effects of computer reminders on complications of peripheral venous catheters and nurses' adherence to a guideline in paediatric care--a cluster randomised study. Implement Sci 2016;11:10.

35 Fossum M, Alexander GL, Ehnfors M, et al. Effects of a computerized decision support system on pressure ulcers and malnutrition in nursing homes for the elderly. Int J Med Inform 2011;80:607-17.

36 Geurts D, de Vos-Kerkhof E, Polinder S, et al. Implementation of clinical decision support in young children with acute gastroenteritis: a randomized controlled trial at the emergency department. Eur J Pediatr 2017;176:173-81.

37 Hovorka R, Kremen J, Blaha J, et al. Blood glucose control by a model predictive control algorithm with variable sampling rate versus a routine glucose management protocol in cardiac surgery patients: a randomized controlled trial. J Clin Endocrinol Metab 2007;92:2960-4.

38 Kroth PJ, Dexter PR, Overhage JM, et al. A computerized decision support system improves the accuracy of temperature capture from nursing personnel at the bedside. AMIA Annu Symp Proc 2006:444-8.

39 Lattimer V, George S, Thompson F. Safety and effectiveness of nurse telephone consultation in out of hours primary care: randomised controlled trial. British Medical Journal 1998;317:1054-9.

40 Lattimer V, Sassi F, George S, et al. Cost analysis of nurse telephone consultation in out of hours primary care: evidence from a randomised controlled trial. BMJ 2000;320:1053-7.

41 Lee N-J, Chen ES, Currie LM, et al. The effect of a mobile clinical decision support system on the diagnosis of obesity and 
overweight in acute and primary care encounters. ANS Adv Nurs Sci 2009;32:211-21.

42 Mann EA, Jones JA, Wolf SE, et al. Computer decision support software safely improves glycemic control in the burn intensive care unit: a randomized controlled clinical study. J Burn Care Res 2011;32:246-55.

43 McDonald MV, Feldman PH, Barron-Vaya Y. Outcomes of clinical decision support (CdS) and correlates of CdS use for home care patients with high medication regimen complexity: a randomized trial. Journal of Evaluation in Clinical Practice 2017;22:10-19.

44 Plank J, Blaha J, Cordingley J, et al. Multicentric, randomized, controlled trial to evaluate blood glucose control by the mode predictive control algorithm versus routine glucose management protocols in intensive care unit patients. Diabetes Care 2006;29:271.

45 Rood E, Bosman RJ, van der Spoel Jl, et al. Use of a computerized guideline for glucose regulation in the intensive care unit improved both guideline adherence and glucose regulation. J Am Med Inform Assoc 2005;12:172-80.

46 Roukema J, Steyerberg EW, van der Lei J, et al. Randomized trial of a clinical decision support system: impact on the management of children with fever without apparent source. J Am Med Inform Assoc 2008:15:107-13.

47 Sassen B, Kok G, Schepers J, et al. Supporting health care professionals to improve the processes of shared decision making and self-management in a web-based intervention: randomized controlled trial. J Med Internet Res 2014;16:e211.

48 Snooks HA, Carter B, Dale J, et al. Support and assessment for fall emergency referrals (safer 1 ): cluster randomised trial of computerised clinical decision support for paramedics. PLoS One 2014;9:e106436.

49 Vadher BD, Patterson DL, Leaning M. Comparison of oral anticoagulant control by a nurse-practitioner using a computer decision-support system with that by clinicians. Clin Lab Haematol 1997;19:203-7.
50 Wells B. Implementation of computerised clinical decision support (CCDS) in a prehospital setting: processes of adoption and impact on Paramedic role and practice. United Kingdom: Swansea University, 2013.

51 Dykes PC, Carroll DL, Hurley A, et al. Fall prevention in acute care hospitals: a randomized trial. JAMA 2010;304:1912-8.

52 Paulsen MM, Paur I, Gjestland J, et al. Effects of using the MyFood decision support system on hospitalized patients' nutritional status and treatment: a randomized controlled trial. Clin Nutr 2020;39:3607-17.

53 Lv S, Ye X, Wang Z, et al. A randomized controlled trial of a mobile application-assisted nurse-led model used to improve treatment outcomes in children with asthma. J Adv Nurs 2019;75:3058-67.

54 Dykes PC, Burns Z, Adelman J, et al. Evaluation of a patientcentered fall-prevention tool kit to reduce falls and injuries: a nonrandomized controlled trial. JAMA Netw Open 2020;3:e202 5889-e89.

55 National Institute for Health and Clinical Excellence (NICE). Guide to the methods of technology appraisal London, 2013. Available: https://www.nice.org.uk/process/pmg9/chapter/the-appraisal-of-theevidence-and-structured-decision-making [Accessed 18 Nov 2020].

56 Murray E, Treweek S, Pope C. Normalisation process theory: a framework for developing, evaluating and implementing complex interventions. BMC Med 2010;8:1-11.

57 Greenhalgh T, Wherton J, Papoutsi C, et al. Beyond adoption: a new framework for theorizing and evaluating nonadoption, abandonment, and challenges to the scale-up, spread, and sustainability of health and care technologies. J Med Internet Res 2017;19:e367.

58 Kawamoto K, McDonald CJ. Designing, conducting, and reporting clinical decision support studies: recommendations and call to action. Ann Intern Med 2020;172:S101-9.

59 National Health Services (NHS). Digital Health: NHSX launches assessment criteria for digital health tools 2020 [cited discussion. Available: https://www.digitalhealth.net/2020/10/nhsx-launchesassessment-criteria-for-digital-health-tools/ [Accessed 29 Oct 2020]. 